\title{
Do practicing primary care physicians believe more complementary and alternative medicine (CAM) training in medical school and residency would have been useful in their current practice?
}

\author{
Michael Malone ${ }^{1,2 *}$ and Gary Tsai ${ }^{1,3}$ \\ ${ }^{1}$ Penn State Hershey Department of Family and Community Medicine, USA \\ ${ }^{2}$ Penn State College of Medicine, USA \\ ${ }^{3}$ Penn State Family and Community Medicine Residency, USA
}

\begin{abstract}
There have been multiple studies performed on attitudes and use of CAM treatments among students, residents, and practicing physicians [1-6]. Attitude towards CAM treatment and education, however, is not the same as utility Therefore, this article reviews the result of a survey that not only looked at the attitudes and use of CAM by practicing physicians, but looked at whether they thought CAM education would have been useful retrospectively in their medical school and residency training.

The results of the survey showed that CAM education during medical school and residency was uncommon among academic primary care attending physicians and most also lacked comfort counseling about CAM treatment and referring patients for CAM therapy. Despite the minimal CAM education in medical school and residency training, the majority of physicians (62\%) felt that CAM education during both medical school and residency would have been helpful in his or her current practice. Therefore, exposing doctors or doctors-in-training to evidence-based information regarding CAM might improve overall clinical care and integration. Future studies are needed to determine if CAM education in medical school or residency training improves physician comfort with CAM therapy counseling and referrals or if it leads to better patient satisfaction or clinical care.
\end{abstract}

\section{Introduction}

There have been multiple studies performed on attitudes and use of CAM treatments among students, residents, and practicing physicians [1-6]. For example, a survey was performed at Mayo Clinic that looked at physicians attitudes (positive/negative) toward CAM in general and their knowledge regarding specific CAM therapies [2]. Surveys have also been performed to determine the desire medical students and residents have to learn about CAM $[3,7,8]$. Medical students have also reported the least amount of education about CAM and viewed CAM therapies as less useful than non-physician health profession students $[7,8]$. CAM education, when incorporated, has often been generally well received in medical school curriculum $[3,9]$.

Attitude towards CAM treatment and education, however, is not the same as utility. In fact, studies have shown that educational exposure to CAM was correlated with attitude towards and the perceived usefulness of CAM [7]. In one study, as high as $90 \%$ of medical students see the need for the integration of CAM into patient care [10]. Unfortunately, medical students and residents perceptions of what is useful for their future practice may not be accurate. Therefore, we created a survey that not only looked at the attitudes and use of CAM by practicing physicians, but looked at whether they thought CAM education would have been useful retrospectively in their medical school and residency training.

\section{Methods}

The survey was sent via email and completed by practicing physicians at an academic center (Penn State) in the departments of Family Medicine, Medicine, and Med-Peds. Thirty-four total responses were obtained. Fourty-one percent of the total responses were from Internal Medicine, thirty-one percent from Med-Peds, and twentyeight percent from Family Medicine (Figure 1). The following questions were asked in the survey and ranked on a likert scale from 0-10:

What is your specialty?

How much exposure did you have to complementary and alternative medicine (CAM) during residency and medical school training?

How would you rate your knowledge of CAM treatments?

Correspondence to: Michael Malone, Penn State Hershey Department of Family and Community Medicine, USA; Penn State College of Medicine, USA, Tel: $717-$ 531-8181; E-mail: mmalone@hmc.psu.edu

Key words: complementary and alternative medicine education, medical school, medical residency, training, primary care, current practice, CAM, training

Received: February 02, 2017; Accepted: February 22, 2017; Published: February 25,2017 
Malone M (2017) Do practicing primary care physicians believe more complementary and alternative medicine (CAM) training in medical school and residency would have been useful in their current practice?

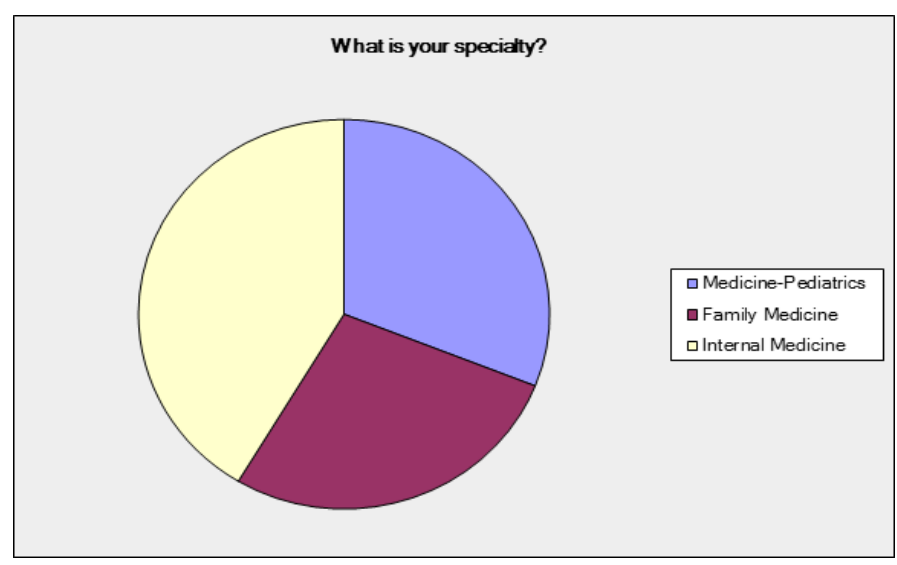

Figure 1. Breakdown of survey responses by specialty.

How much exposure to Complementary and Alternative Medicine did you have, if any, during medical schooll or residency training?

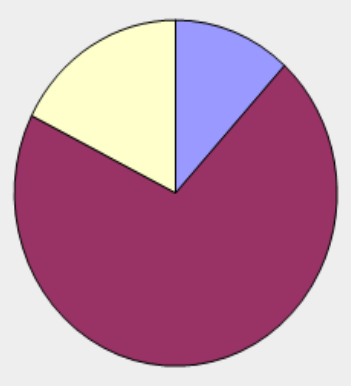

口None at all

aMinimal amount

口Moderate amount

口Significant amount

-Extensive amount

Figure 2. Perceived exposure to CAM education during medical school and residency.

How likely are you to refer a patient with a non-emergent condition for CAM treatment?

Please rate your level of comfort counseling a patient on CAM therapies?

Do you think more exposure to CAM (short lectures, hands-on sessions, seminars) during your medical school education would have been beneficial to your current practice?

Do you think more exposure to CAM (short lectures, hands-on sessions, seminars) during your residency education would have been beneficial to your current practice?

\section{Results}

A baseline assessment of physician comfort level counseling patients on CAM therapy showed very low comfort with an average of 3.75/10 on a ten-point Likert scale. Only twenty-eight percent of the physicians noted that they felt comfortable referring patients for CAM treatment. Results of the survey showed very little exposure to CAM education during medical school and residency with $82 \%$ of physicians noting minimal or no exposure at all (Figure 2). Despite lack of exposure to CAM education, the majority of physicians (62\%) felt that CAM education during both medical school and residency would have been helpful in his or her current practice (Figure 3 and Figure 4).

\section{Limitations}

Small sample size due is a limiting factor as well as possible different

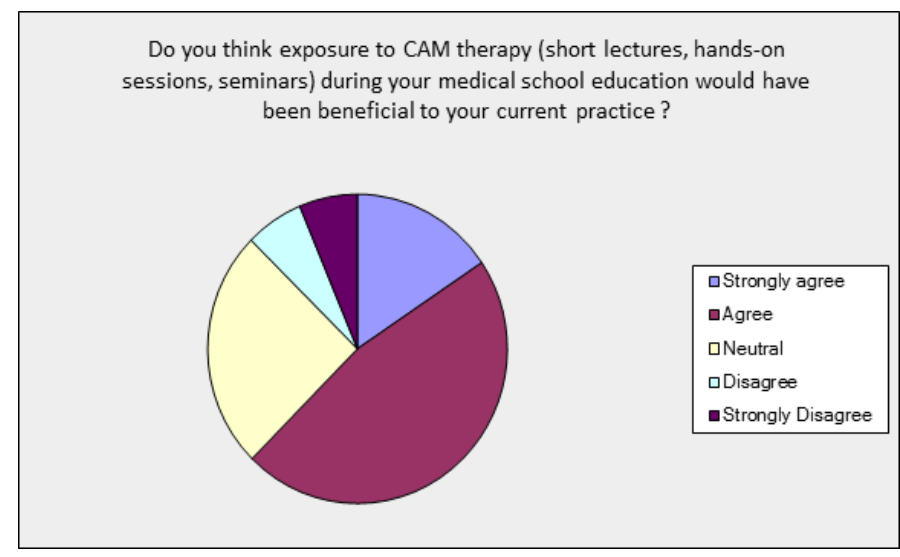

Figure 3. Perceived Utility of CAM Exposure In Medical School Education.

Do you think exposure to CAM therapy (short lectures, hands-on sessions, seminars) during your residency education would have been beneficial to your current practice?

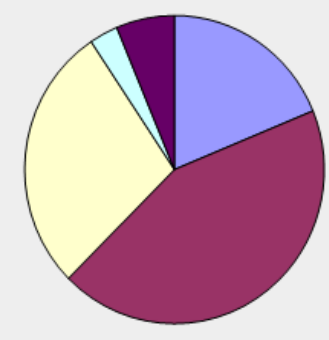

口Strongly agree

aAgree

$\square$ Neutral

口Disagree

- Strongly Disagree

Figure 4. Perceived Utility of CAM Exposure In Residency Education.

interpretations of questions by each survey responder.

\section{Conclusion}

CAM education during medical school and residency was uncommon among academic primary care attending physicians in our survey and most also lacked comfort counseling about CAM treatment and referring patients for CAM therapy. This implies that the discomfort in both referring and counseling patients on CAM treatment is likely related to the paucity of CAM education in medical school and residency. Therefore, exposing doctors or doctors-intraining to evidence-based information regarding CAM might improve overall clinical care and integration.

Despite the minimal CAM education in medical school and residency training, the majority of physicians (62\%) felt that CAM education during both medical school and residency would have been helpful in his or her current practice. These results suggest that CAM education likely needs to be increased in both medical school and residency. Future studies are needed to determine if CAM education in medical school or residency training improves physician comfort with CAM therapy counseling and referrals or if it leads to better patient satisfaction or clinical care.

\section{References}

1. Roy V, Gupta M, Gosh RK (2015) Perception, attitude and usage of complementary and alternative medicine among doctors and patients in a tertiary care hospital in India. Indian J Pharmacol 47: 137-142.

2. Wahner-Roedler DL, Vincent A, Elkin PL, Loehrer LL, Cha SS, et al. (2006) Physicians' 
Malone M (2017) Do practicing primary care physicians believe more complementary and alternative medicine (CAM) training in medical school and residency would have been useful in their current practice?

attitudes toward complementary and alternative medicine and their knowledge of specific therapies: a survey at an academic medical center. Evid Based Complement Alternat Med 3: 495-501.

3. Damgaard-Mørch NL, Nielsen LJ, Uldall SW (2008) [Knowledge and perceptions of complementary and alternative medicine among medical students in Copenhagen] Ugeskr Laeger 170: 3941-3945. [Crossref]

4. Brokaw JJ, Tunnicliff G, Raess BU, Saxon DW (2002) The teaching of complementary and alternative medicine in U.S. medical schools: a survey of course directors. Acad Med 77: 876-881. [Crossref]

5. Wetzel MS, Kaptchuk TJ, Haramati A, Eisenberg DM (2003) Complementary and alternative medical therapies: implications for medical education. Ann Intern Med 138 : 191-196. [Crossref]

6. Zimmerman C, Kandia J (2012) A Pilot Study to Assess Students' Perceptions,
Familiarity, and Knowledge in the Use of Complementary and Alternative Herbal Supplements in Health Promotion. Alternative Therapies 18: 5.

7. Baugniet J, Boon H, Ostbye T (2000) Complementary/alternative medicine: comparing the view of medical students with students in other health care professions. Fam Med 32: $178-184$.

8. Burman ME (2003) Complementary and alternative medicine: core competencies for family nurse practitioners. $J$ Nurs Educ 42: 28-34. [Crossref]

9. Halterman-Cox M, Sierpina VS, Sadoski M, Sanders C (2008/2009) CAM Attitudes in First- and Second-year Medical Students: A Pre- and Post-course Survey Integrative Medicine 7: 6.

10. Tiralongo E, Wallis M (2008) Attitudes and perceptions of Australian pharmacy students towards Complementary and Alternative Medicine - a pilot study. BMC Complement Altern Med 8: 2. [Crossref]

Copyright: (C2017 Malone M. This is an open-access article distributed under the terms of the Creative Commons Attribution License, which permits unrestricted use, distribution, and reproduction in any medium, provided the original author and source are credited. 